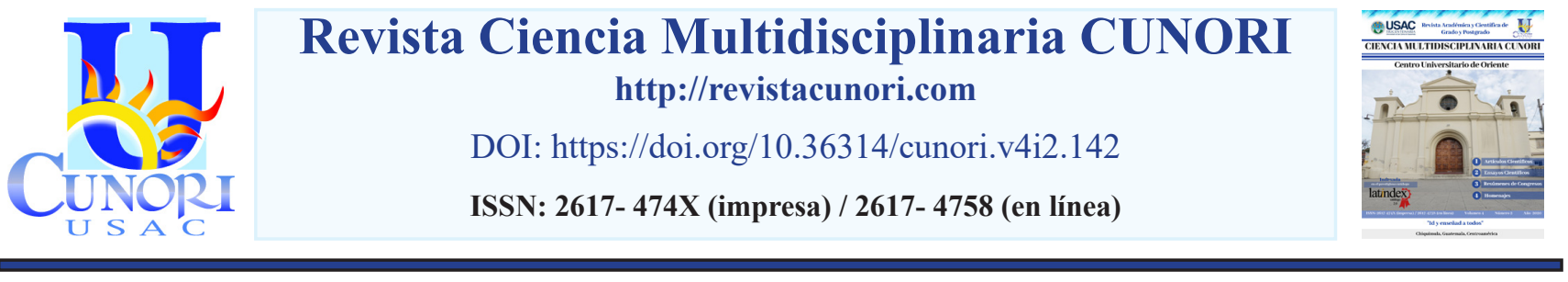

Referencia del resumen de congreso

Mosquera-Saravia M. T. (2020). III Congreso de la sociedad de antropología médica de la Asociación Americana de antropología. Revista Ciencia Multidisciplinaria Cunori, 4(2), 171-178. https://doi.org/10.36314/cunori.v4i2.142

\title{
III Congreso de la sociedad de antropología médica de la Asociación Americana de Antropología
}

\section{Congress of the Medical Anthropology Society of the American Anthropological Association}

\author{
María Teresa Mosquera Saravia \\ Universidad de San Carlos de Guatemala \\ https://orcid.org/0000-0003-0924-0302 \\ La Habana, Cuba del 9 al 12 de marzo 2020 \\ Recibido: 13 de abril de 2020 / Revisado: 20 de abril de 2020 / Aceptado: 29 de mayo de 2020 \\ Disponible en internet el 30 de octubre de 2020
}

\author{
*Autor para correspondencia. \\ Resumen \\ Correo electrónico: teresa.mosquera@usac.edu.gt
}

Z1 III Congreso de la sociedad de antropología médica de la Asociación Americana de Antropología, se realizó en La Habana, ECuba del 9 al 12 de marzo 2020. La Doctora María Teresa Mosquera Saravia compiló los resúmenes del congreso de la mesa expositora. Para la actividad es establecieron tres objetivos: 1. Brindar los antecedentes históricos regionales sobre las acciones de salud intercultural desarrolladas en América latina en la segunda mitad del siglo XX y el presente. 2. Describir y analizar -de manera crítica- las políticas públicas sobre salud intercultural que se han generado por los gobiernos de la Región. 3. Presentar los recientes avances, estancamientos y retrocesos en proyectos de investigación, programas docentes y actividades sanitarias relacionadas con la salud intercultural en América Latina, centrados en los pueblos originarios y afrodescendientes. Participaron importantes expertos como los doctores Roberto Campos Navarro. Miguel Güémez con la ponencia Salud, enfermedad y alimentación durante el embarazo en el contexto intercultural: un estudio de caso con mujeres mayas del oriente de Yucatán. Gina Carrioni con la ponencia ¿salud intercultural, servicios de salud adecuados socioculturalmente, modelos o sistemas de salud propios e interculturales? la experiencia en la formulación de una política pública en salud para los pueblos indígenas de Colombia. Noly Fernández con el título de ponencia A 15 años de interculturalidad en salud en Venezuela. María Teresa Mosquera Saravia con la ponencia Interculturalidad de la salud en Guatemala. Romano González con la ponencia Avances y retrocesos en la política sanitaria para pueblos indígenas en Costa Rica: ¿sobrevivirá el modelo de salud indígena? Lucy Orellana de Piscoya Parto vertical quechua: fundamentos de la lógica científica en comunidades andinas Acocro y Pampamarca de Ayacucho 2013. Margarita Sáez Salgado con la ponencia El puente roto de la salud intercultural en chile. Roberto Campos Navarro con la ponencia Treinta años de salud intercultural en poblaciones indígenas de México: un proceso incompleto e inacabado.

Palabras clave: pueblos indígenas, salud intercultural

Abstract

$\mathrm{T}$ he III Congress of the medical anthropology society of the American Anthropological Association was held in Havana, Cuba from March 9 to 12, 2020. Dr. María Teresa Mosquera Saravia compiled the summaries of the congress of the exhibiting table. For the activity, three objectives were established: 1. Provide regional historical background on intercultural health actions developed in Latin America in the second half of the 20th century and the present. 2. Describe and analyze -critically- the public poli- 
cies on intercultural health that have been generated by the governments of the Region. 3. Present the recent advances, stagnations and setbacks in research projects, educational programs and health activities related to intercultural health in Latin America, focused on indigenous peoples and Afro-descendants. Important experts such as doctors Roberto Campos Navarro participated. Miguel Güémez with the presentation Health, illness and diet during pregnancy in the intercultural context: a case study with Mayan women from eastern Yucatán. Gina Carrioni with the presentation intercultural health, socioculturally appropriate health services, own and intercultural health models or systems? the experience in the formulation of a public health policy for the indigenous peoples of Colombia. Noly Fernández with the title of presentation A 15 years of interculturality in health in Venezuela. María Teresa Mosquera Saravia with the presentation: Interculturality of health in Guatemala. Romano González with the presentation Advances and setbacks in health policy for indigenous peoples in Costa Rica: will the indigenous health model survive? Lucy Orellana from Piscoya Quechua vertical birth: foundations of scientific logic in Andean communities Acocro and Pampamarca de Ayacucho 2013. Margarita Sáez Salgado with the presentation The broken bridge of intercultural health in Chile. Roberto Campos Navarro with the presentation Thirty years of intercultural health in indigenous populations of Mexico: an incomplete and unfinished process.

Keywords: indigenous populations, intercultural health

\section{Ponencia presentada por el Doctor Roberto Campos Navarro Contenido}

Es Doctor en Antropología y Médico de la Universidad Nacional Autónoma de México de la Facultad de Medicina del Departamento de Historia de la Medicina. En la década de los cincuenta del siglo pasado, en América latina se inician los esfuerzos por incorporar cambios e innovaciones en la salud de los pueblos originarios. En este contexto resultó relevante el texto denominado "Los programas de salud en la situación intercultural" (1955), del médico y antropólogo mexicano Gonzalo Aguirre Beltrán, en el cual expuso las bases fundamentales de la atención sanitaria con enfoque intercultural. Serían los Centros Coordinadores del Instituto Nacional Indigenista (INI), establecidos en las más importantes regiones indígenas del país (Chiapas, Michoacán, Oaxaca, Yucatán, etc.), quienes pondrían en marcha algunos de los postulados del Dr. Aguirre Beltrán. En Chile surgirían programas interculturales desde 1994 con la creación de las oficinas de orientación intercultural (denominadas Amuldungún) en las regiones indígenas mapuches al sur chileno, que luego se extendería -con reconocibles alcances- al resto del país. Con desiguales resultados, se implantarían oficinas semejantes en Bolivia (Willaqkuna en Potosí), en el Perú (Ayacucho) y sobre todo, con demostrada eficacia en Venezuela durante el gobierno del presidente Hugo Chávez. Otros países que han establecido programas de salud intercultural serían Colombia, Guatemala, Costa Rica y Panamá. A poco más de 60 años del inicio de prácticas sanitarias en pueblos originarios, afrodescendientes y comunidades rurales, consideramos conveniente hacer un balance de las fortalezas y debilidades detectadas con estas políticas gubernamentales relacionadas con la salud intercultural.

Objetivos: 1. Brindar los antecedentes históricos regionales sobre las acciones de salud intercultural desarrolladas en América latina en la segunda mitad del siglo XX y el presente. 2. Describir y analizar-de manera crítica- las políticas públicas sobre salud intercultural que se han generado por los gobiernos de la Región. 3. Presentar los recientes avances, estancamientos y retrocesos en proyectos de investigación, programas docentes y actividades sanitarias relacionadas con la salud intercultural en América Latina, centrados en los pueblos originarios y afrodescendientes. 


\section{Ponencia presentada por el Doctor Miguel Güémez}

Es Doctor en Antropología de la Universidad Autónoma de Yucatán, México presentó la ponencia La salud, enfermedad y alimentación durante el embarazo en el contexto intercultural: un estudio de caso con mujeres mayas del oriente de Yucatán.

\section{Contenido}

Este trabajo presenta los resultados preliminares del proyecto colectivo "Alimentación, salud y enfermedad durante el embarazo en el contexto intercultural: el caso de mujeres mayas con sobrepeso y obesidad en Yaxcaba, Yucatán (2018-2020)" que se desarrolla con una metodología de acción participativa, y cuyo propósito es construir, a partir de un diagnóstico, un Programa de lntervención Alimentaria con enfoque intercultural e interdisciplinario para el mejoramiento cualitativo de la nutrición en el Primer Nivel de Atención durante el embarazo-puerperio que coadyuve a la prevención de enfermedades y complicaciones asociadas: abortos, partos prematuros, preeclampsia, enfermedades crónico degenerativas. Este Programa prevé una alimentación y nutrición sustentable, intercultural y participativa que articule elementos de la biomedicina y de las tradiciones, saberes y productos locales. Estará basado de guías de acompañamiento diseñadas con la información surgida en los diálogos (grupos focales) entre las mujeres, las parteras/os locales y el personal del Centro de Salud, y en general, de los resultados y productos obtenidos en el proyecto. En este contexto nos hemos propuesto: 1) Propiciar un proceso de interacción donde el conocimiento académico se articule con los saberes locales del pueblo maya y del personal médico. 2) Facilitar y crear las condiciones en que esa colaboración e interacción debería darse u ocurrir. 3) Delimitar las aportaciones de los académicos (antropólogos, estudiantes y profesores de ciencias de la salud); los compromisos de los representantes del sector salud, así como las aportaciones de los representantes del pueblo maya; 4) ldentificar, en el proceso, las fortalezas, dificultades y limitaciones del llamado enfoque intercultural y el diálogo de saberes. Finalmente, el proyecto busca que este diálogo de saberes sea un paradigma colaborativo para el ejercicio de una interculturalidad adecuada y pueda resolver problemas de alimentación en el campo de la salud reproductiva.

\section{Ponencia de la Doctora Gina Carrioni}

Representante de la Universidad de los Andes, Colombia, presentó la ponencia Salud intercultural, servicios de salud adecuados socioculturalmente, modelos o sistemas de salud propios e interculturales, la experiencia en la formulación de una política pública en salud para los pueblos indígenas de Colombia.

\section{Contenido}

Análisis de la normatividad -nacional e internacional-y políticas públicas que posibilitan en Colombia la estructuración de un Sistema de Salud Propio e Intercultural de manera participativa con y para los pueblos indígenas. Situación de salud de los pueblos indígenas - desde los datos oficiales y desde las problemáticas identificadas por los pueblos indígenas- que justifica la estructuración de un sistema de salud diferente para los pueblos indígenas. Concepciones frente a la salud intercultural, salud propia, medicina tradicional o ancestral, adecuación sociocultural, modelos de salud, sistema de salud propio e intercultural -principios rectores, componentes, actores-, participación, consulta y concertación en 
salud, promoción y prevención en salud, acciones individuales y colectivas, entre otros, desde la perspectiva institucional -gubernamental nacional y local, de las ONG y organismos internacionales- y desde la de los pueblos indígenas y sus organizaciones. Cómo surge la iniciativa de un sistema de salud propio e intercultural para los pueblos indígenas y su relación o articulación con el Sistema de Salud colombiano, cómo se ha venido estructurando, sus avances, estancamientos, retrocesos y retos, quiénes han participado en su elaboración y cómo se ha dado esa participación, roles y responsabilidades de los actores participantes del proceso y de los demás sectores que inciden en la salud -intersectorialidad-, cómo se propone operativizar en el territorio.

\section{Ponencia presentada por Noly Fernández}

\section{De la Universidad de las Ciencias de la Salud Hugo Chávez Frías, Venezuela. Pre- sentó la ponencia A 15 años de interculturalidad en salud en Venezuela.}

\section{Contenido}

De acuerdo con el marco jurídico vigente que a partir del año 2004 se crea en el Ministerio del Poder Popular para la Salud la Coordinación de Salud Indígena (hoy Dirección General de Salud Intercultural) con la misión de formular y evaluar políticas en salud en el marco de un enfoque intercultural con pueblos y comunidades indígenas, a fin de contribuir a transversalizar el enfoque intercultural, generando respuestas oportunas, pertinentes, sostenibles y potentes al imperativo ético-político de las necesidades sociales de pueblos y comunidades indígenas que el Estado venezolano está comprometido a desarrollar. Estas series de acciones y las respuestas de apoyos a los hospitales, personal de salud y las comunidades indígenas permitieron el crecimiento y la extensión de los Servicios de Atención y Orientación al Indígena (SAOI) en las distintas regiones del país con presencia indígena; estrategia que permitió implementar otros proyectos de gran éxito como el Plan de Salud Yanomami (PSY), Proyecto Cacique Nigale (PCN), Programa de Apoyo a las Poblaciones Warao del Delta del Orinoco (Prowarao), Plan Apure (PSA), Programas de Formación y Sensibilización en Interculturalidad en Salud, entre otros. Tomando en cuenta lo anteriormente expuesto esta dirección ejecuta sus acciones desde hace aproximadamente 15 años a nivel nacional.

Dentro de las limitaciones del sistema de salud, el MPPS ha enfrentado una serie de dificultades históricas, económicas, sociales, culturales y políticas que han sido obstáculos para el fortalecimiento del Sistema Público de Salud con enfoque intercultural. Aún cuando se ha impulsado diferentes estrategias para fortalecer los servicios públicos de salud, los esfuerzos no han logrado su objetivo. Si bien hay una escasez importante de recursos financieros, también existen otros problemas como la forma en que se comprende la salud-enfermedad, como fortalecer la medicina tradicional indígena, la forma en que se organiza la atención, la formación y gestión del personal de salud, la generación de información y conocimiento, y la administración de otros recursos. 


\section{Ponencia presentada por la Doctora María Teresa Mosquera Saravia}

\section{Del Instituto de Estudios Interétnicos y de los Pueblos Indígenas de la Universidad de San Carlos de Guatemala es Doctora en Antropología Social y Cultural, presentó la ponencia Interculturalidad de la salud en Guatemala.}

\section{Contenido}

La interculturalidad de la salud es un tema que se ha implementado muy lentamente en Guatemala, las ONGS han sido las instituciones pioneras de la implementación de programas interculturales de la salud, en algunas comunidades su impacto ha sido tan grande que han logrado disminuir la mortalidad materna. El Ministerio de Salud Pública y Asistencia Social -MSPAS-, se ha preocupado de implementar algunas políticas con pertinencia cultural, entre ellas: el parto vertical, el uso de los idiomas mayas, construcciones de temazcales o tuj, etc. Uno de los aportes más importantes fue la implementación del Modelo Incluyente de Salud -MIS-, este programa de atención primaria en salud, se ocupa de 4 ejes: género, medio ambiente, interculturalidad y derecho a la salud. Sin embargo, esta propuesta de atención a la salud primaria, no pudo prolongarse en el tiempo, debido a diversos problemas. En una sociedad como la guatemalteca la falta de formación en el tema de la cosmovisión es lo que provoca un atropello, una falta de comprensión y de entendimiento del otro, razón por la que el tema de la interculturalidad de la salud es un factor indispensable en la recuperación de la salud del paciente y en la creación de una política con pertinencia cultural.

\section{Ponencia presentada por Romano González}

\section{Del Ministerio de Salud, Costa Rica. Presentó la ponencia Avances y retrocesos en la política sanitaria para pueblos indígenas en Costa Rica: ¿sobrevivirá el modelo de salud indígena?}

\section{Contenido}

Costa Rica tuvo sobre todo durante la década de 1970 avances sobresalientes en sus indicadores de salud. Sin embargo, en el Censo de Población del año 2000 la pirámide de población para los Pueblos Indígenas (que son un 2,4\% de la población total, era la de la Costa Rica de 1970, es decir, había un rezago de 30 años para la población indígena, solo explicable por las diferencias culturales entre éstos y la "cultura" del Estado, que no supieron manejarse para respetar las primeras y en términos generales, se procuraron borrar las diferencias imponiendo el modelo occidental de prevención y atención de la enfermedad. Pese a ello, sobrevive en muchos territorios indígenas (son 24 en el país) un fuerte arraigo cultural, incluyendo la presencia de "médicos" indígenas. El cambio cultural sin embargo es rápido y ubicuo. Las instituciones prestadoras de servicios en salud han procurado ajustes. El más temprano fue el de la formación de Asistentes Técnicos de Atención Primaria en Salud procedentes de Territorios Indígenas, pero en el marco de la medicina occidental. En 2003 se desarrollo una dinámica particular y surgió la $1^{\circ}$ Política en Salud para Pueblos Indígenas. En 2006 se conformó el Consejo Nacional de Salud de Pueblos Indígenas para dar seguimiento a la estrategia. Recientemente se han integrado profesionales indígenas en medicina graduados en Cuba al sistema de salud. Por razones burocráticas no se cuenta con un Plan Nacional de Salud para Pueblos Indígenas. En todo esto....¿está sobreviviendo el modelo de salud indígena? El cambio cultural es rápido y ubicuo y las acciones estatales puntuales y lentas. Apenas en 2015 se incorpora a la Constitución Política de la República el reconocimiento de su carácter multiétnico y pluricultural. Si se trata de sumar lo mejor de las culturas occidental e indígena 
para la prevención, promoción y atención de la enfermedad y de que sobreviva el modelo de salud indígena (alimentos, agricultura, plantas medicinales, curadores, espiritualidad), el futuro no es muy halagüeño. Sin embargo, como muestra de las capacidades institucionales, en 2018 se oficializa el $1^{\circ}$ Plan Nacional de Salud para Afrodescendientes.

\section{Ponencia de la doctora Lucy Orellana de Piscoya}

De la Universidad Nacional Federico Villarreal, Perú, presentó la ponencia Parto vertical quechua: fundamentos de la lógica científica en comunidades andinas Acocro y Pampamarca de Ayacucho 2013.

\section{Contenido}

El Parto Vertical Andino Quechua acuna saberes y practicas ancestrales, actualmente es de uso cotidiano en comunidades andinas de Perú. La legitimidad social y seguridad cultural hacen persistir en tiempo y espacio demostrando lógica y coherencia basado en fundamentos científicos que otorgan validez desde ámbitos: Históricos a través de iconografías, idioma quechua que patrocina comunicación en el proceso y descripción de la toponimia en la estructura anatómica y fisiología del parto. Culturales: es parte de la medicina tradicional basada en la cosmovisión andina, que otorga presupuestos tangibles y no tangibles lógicos para la atención. Filosóficos: evidencia saberes y prácticas asociadas a principios andinos de "reciprocidad", "relacionalidad", "armonía" y "complementariedad" en comportamientos de la partera, parturienta, familiares. Endocrinológicos: valora aspectos de la voluntad y el accionar instintivo de la mujer; "neocortex" en reposo no admite miedo, las estructuras primitivas hipotálamo e hipófisis desencadenan mayor secreción oxitócica por ambiente seguro, con acompañamiento, sin bulla, libertad de la mujer para elegir posición y dirigir su parto, situaciones que atenúan la activación del cerebro de la racionalidad. Físico: reconoce puntos de apoyo, alimentación, masajes manejo de frio/ calor, uso de fitoterapia. Psicológico/espiritual: prioridad en el mundo andino, uso del "ritual" como medio para alcanzar y valorar el equilibrio psicosomático. Conclusión: Existe Lógica y coherencia en los saberes y prácticas de la atención del parto vertical quechua y los fundamentos descritos que otorgan argumento y validez científica. Estudio cualitativo alcanzado con la participación de parteras, parejas y familias de la comunidad de Pampamarca en Ayacucho Perú.

\section{Ponencia presentada por la Doctora Margarita Sáez Salgado \\ De la Universidad de Chile, presentó la ponencia El puente roto de la salud inter- cultural en chile.}

\section{Contenido}

Muchos se han preguntado por qué Chile tiene una historia que compartir en salud y pueblos indígenas bajo el concepto de la controvertida interculturalidad en salud, el supuesto básico es que constituyen un bajo porcentaje de la población. Porqué tienen más de 20 años de trabajo con presupuesto asignado al Programa Nacional de Salud y Pueblos Indígenas y tantas experiencias conocidas en otros países. Tratar de responder estás y muchas otras preguntas es el objetivo de esta ponencia. Desde una mirada al contexto, primero reflexionar sobre el proceso de construcción de una política de salud indígena en el sector salud considerando un Estado cooptado por el neoliberalismo en los gobiernos de la posdictadura, cómo fue posible hacerlo con participación y en el escenario de una Ley Indígena que no menciona a salud y que no habla de pueblos. Principalmente, aportar una aproximación al papel de los antropólogos en 
este complejo proceso, ilustrar los acuerdos y desacuerdos sobre interculturalidad, modelos de atención complementarios, reconocimiento de los sistemas de salud de los pueblos, formación y capacitación de recursos humanos, conceptos de salud-enfermedad-muerte, epidemiología sociocultural. Al finalizar, compartir cómo se implementó el proceso Nacional de Consulta y Participación del Reglamento para el Artículo 7 de la de Ley de Derechos y Deberes en Atención de Salud, sobre Interculturalidad en la Red de Servicios, realizado bajo los principios del Convenio 169 de la OIT, el que duró al menos tres años y su estado actual.

\section{Ponencia presentada por el Doctor Roberto Campos Navarro}

Es Doctor en Antropología y Médico de la Universidad Nacional Autónoma de México de la Facultad de Medicina del Departamento de Historia de la Medicina. Presentó la ponencia Treinta años de salud intercultural en poblaciones indígenas de México: un proceso incompleto e inacabado.

\section{Contenido}

A finales de la década de los ochenta, el gobierno mexicano firmó y ratificó el Convenio 169 de la Organización Internacional del Trabajo, cuyo artículo 25 versa sobre el reconocimiento de la medicina tradicional y la participación de los pueblos indígenas en la planificación de los servicios de salud en sus comunidades. El Instituto Nacional Indigenista -durante el periodo 1990-1996- cumplió un papel apreciable al relacionarse de manera muy cercana con las organizaciones de médicos indígenas de todo el país, incorporando una organización al interior del único hospital administrado por la institución y creando la primera biblioteca sobre la medicina tradicional con quince tomos que describen el estado de sus recursos humanos, materiales y simbólicos. El Instituto Mexicano del Seguro Social -en su versión rural- consolida su programa de interrelación con la medicina indígena en las áreas donde tiene presencia institucional, pero no fue sino hasta inicios del presente siglo, que la Secretaría de Salud inicia una política de salud intercultural a nivel nacional, con un limitado ejercicio normativo, una notable precariedad de actividades operativas y un reducido apoyo presupuestal. Todo ello se refleja en una atención de la red hospitalaria (en regiones indígenas) carente de una verdadera funcionalidad en términos interculturales.

\section{Referencias}

Mosquera-Saravia M. T. (2020). III Congreso de la sociedad de antropología médica de la Asociación Americana de antropología. Revista Ciencia Multidisciplinaria Cunori, 4(2), 179-183. https://doi.org/10.36314/cunori.v4i2.143

\section{Sobre la autora}

\section{María Teresa Mosquera Saravia}

Antropóloga del Instituto de Estudios Interétnicos y de los Pueblos Indígenas de la Universidad de San Carlos de Guatemala. 
Copyright (c) María Teresa Mosquera Saravia

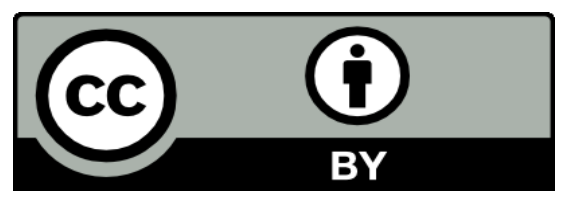

Este texto está protegido por una licencia CreativeCommons 4.0.

Usted es libre para compartir, copiar y redistribuir el material en cualquier medio o formato y adaptar el documento, remezclar, transformar y crear a partir del material para cualquier propósito, incluso comercialmente, siempre que cumpla la condición de atribución: usted debe reconocer el crédito de una obra de manera adecuada, proporcionar un enlace a la licencia, e indicar si se han realizado cambios. Puede hacerlo en cualquier forma razonable, pero no de forma tal que sugiera que tiene el apoyo del licenciante o lo recibe por el uso que hace. 